\title{
Editorials
}

\section{Which first-line antidepressant?}

Choice of first-line antidepressants for depression has been debated in psychiatric journals over the last 9 months, in relation to the widely reported meta-analysis by Cipriani et al in the Lancet, comparing 21 antidepressants for efficacy and tolerability. They found that agomelatine, amitriptyline, escitalopram, mirtazapine, paroxetine, venlafaxine, and vortioxetine were more effective than other antidepressants. They also found agomelatine, citalopram, escitalopram, fluoxetine, sertraline, and vortioxetine to be relatively better tolerated than others. ${ }^{1}$ Three antidepressants with higher efficacy also had relatively high acceptability: agomelatine, escitalopram, and vortioxetine. So should these antidepressants now be considered firstline choices for depression in primary care?

\section{NETWORK META-ANALYSIS}

The Cipriani group's conclusions should be treated with some caution, as they are based on network meta-analysis (NMA). NMA methodology enables multiple treatments to be compared using both direct comparisons within randomised controlled trials, and indirect comparisons across trials based on a common comparator. So, if antidepressants ' $A$ ' and ' $C$ ' have each been compared with antidepressant 'B' directly, you can infer how 'A' would perform compared with ' $C$ ' through NMA, even if ' $A$ ' and ' $C$ ' have never been compared in the same trial. However, the inferences from NMAs that some antidepressants are more effective or acceptable than others are not always consistent with direct headto-head comparisons of drugs within trials. It is therefore important to look also at systematic reviews of trials comparing drugs with each other directly.

This is not the first time Cipriani's group has suggested that escitalopram should be a preferred first-line choice due to its combined higher efficacy and tolerability. In 2009, they published an NMA comparing 12 antidepressants showing similar clinically important differences in favour of escitalopram and sertraline. ${ }^{2}$ Following the 2009 study, sertraline prescribing rose significantly while citalopram prescribing levelled off, and that of fluoxetine fell. ${ }^{3}$ However, escitalopram prescribing did not increase significantly. ${ }^{3}$ That may have been because escitalopram was still under patent and was significantly more expensive, but, since patent expiry, generic escitalopram costs have fallen to match other selective serotonin reuptake inhibitors (SSRIs). ${ }^{4}$

\section{ESCITALOPRAM}

So why not choose escitalopram firstline? One reason is safety concerns. As with citalopram, it can cause significant QTc prolongation, potentially increasing the risk of ventricular arrhythmias, which led to the 2011 Medicines and Healthcare products Regulatory Agency warning, restricting use and doses, ${ }^{5}$ following which citalopram and escitalopram prescribing both fell. ${ }^{3}$ QTc prolongation is less of an issue with sertraline and fluoxetine. Also, some escitalopram comparison studies use low therapeutic doses of escitalopram (for example, $10 \mathrm{mg}$ dailyl and non-equivalent significantly higher comparator SSRI doses (for example, sertraline 200 mg daily), which are known to lack greater efficacy but are associated with poorer tolerance and higher dropout rates. ${ }^{6.7}$ Conversely, comparator studies with less effective low-dose land so non-dual action) venlafaxine (for example, $75 \mathrm{mg}$ daily) may be used to demonstrate and claim equivalent efficacy. ${ }^{6}$ There is still a relative lack of direct head-to-head trial evidence for escitalopram's claimed superiority over other antidepressants, apart from citalopram. ${ }^{6}$

Agomelatine is thought to act through a combination of antagonist activity at 5 HT2C receptors and agonist activity at melatonergic MT1/MT2 receptors, which makes it unique among antidepressants, as it does not affect the reuptake of serotonin, norepinephrine (noradrenaline), or dopamine. A meta-analysis of direct headto-head studies comparing it with SSRIs and serotonin and norepinephrine inhibitors (SNRIs) found that it had similar efficacy, although published trials generally had more favourable results than unpublished trials. ${ }^{8}$ Given that a year's treatment costs significantly more, at $€ 390$ per annum, than fluoxetine ( $€ 7$ ), sertraline ( $€ 10)$, escitalopram (£14), or citalopram (£13), ${ }^{4}$ and it requires liver function monitoring, agomelatine should currently be limited to a third-line choice. However, it may be considered as a viable alternative when SSRIs, SNRIs, and mirtazapine are all contraindicated.

Vortioxetine is a serotonin transporter blocker that increases the extracellular concentration of serotonin, dopamine, and norepinephrine, and so acts like an SNRI. A 2017 Cochrane review found no advantage when it was compared with SNRIs, being less effective than duloxetine, although it had less severe adverse effects. ${ }^{9}$ The review criticised a relative lack of direct head-tohead comparisons between vortioxetine and the SSRIs, and the reliance placed on the results of NMAs to define its role. ${ }^{9}$ Given that a year's treatment costs $€ 360,{ }^{4}$ vortioxetine also should remain a third-line choice.

\section{MIRTAZAPINE}

What about mirtazapine, which Cipriani et al found to be ranked highly for efficacy, but not so highly for acceptability? It is relatively popular: GP prescribing of mirtazapine first-line for both first ever and recurrent episodes of depression has been increasing steadily since $2003,{ }^{3}$ and by 2017 mirtazapine accounted for $12 \%$ of antidepressant prescriptions in England..$^{10} \mathrm{~A} 2011$ Cochrane systematic review of 29 randomised controlled trials comparing mirtazapine directly with other antidepressants found that mirtazapine was superior to SSRIs at the end of initial treatment over 6 to 12 weeks. ${ }^{11}$ Mirtazapine treatment led to a similar frequency of dropouts as SSRIs and tricyclic antidepressants (TCAs), although its adverse event profile was unique, characterised by weight gain and sedation in a significant proportion of patients, but fewer gastrointestinal problems and sexual dysfunction than SSRIs."

So how should GPs choose a first-line antidepressant for major depressive disorder? The 2009 National Institute for Health and Care Excellence guidance ${ }^{12}$ and the British Association for Psychopharmacology $(B A P)^{7}$ suggest an SSRI should be considered first, unless there is a history of poor response or unacceptable side effects with SSRIs.

It is important to emphasise that antidepressant treatment is best avoided at the initial consultation if possible, ${ }^{13}$ and should only be prescribed if psychological interventions or exercise have either been tried first or are thought to be unsuitable, or the patient has recurrent depression and is asking for drug treatment, or the patient is at risk of developing more severe depression (for example, if they have a history of severe depression).

There are relatively few differences between SSRIs, although paroxetine is best 
avoided unless patients particularly ask for it, given its short half-life, which leads to a greater risk of discontinuation symptoms, and its greater tendency to cause sexual dysfunction and weight gain. Sertraline is probably a safer choice than citalopram or escitalopram due to the QTc prolongation issue and their potential interactions with, for example, methadone, antipsychotics, and erythromycin, although it causes more diarrhoea. Important interactions to consider include paroxetine inhibition of tamoxifen; fluoxetine potentiation of the seizure risk with clozapine; and fluvoxamine potentiation of theophylline and clozapine, through inhibition of hepatic cytochrome P450 enzymes.

\section{PROBLEMS WITH THE SSRIS}

SSRIs as a class increase the risk of gastrointestinal, uterine, and cerebral bleeding, particularly when taken with aspirin, non-steroidal anti-inflammatories, or anticoagulants. They should be avoided by patients with increased risks of bleeding, and given together with a protein pump inhibitor for patients with dyspepsia. They are also more likely to cause hyponatraemia, especially for patients taking diuretics. Rarely, concomitant SSRI and tramadol use can lead to serotonin syndrome. For patients with these relative contraindications, mirtazapine, nortriptyline, or lofepramine would be a better first choice. Mirtazapine could be chosen if sedation and stimulation of appetite are desired effects, or else a TCA or TCA-type drug such as nortriptyline or lofepramine, if sedation and weight gain are to be avoided. Mirtazapine should be titrated up from $15 \mathrm{mg}$ daily to at least $30 \mathrm{mg}$, as $15 \mathrm{mg}$ may help anxiety and insomnia symptoms in the short term, but is sub-therapeutic for treating major depression. ${ }^{14}$ SSRIs often cause sexual dysfunction, as do SNRIs and TCAs: mirtazapine, bupropion, moclobemide, agomelatine, and vortioxetine are less likely to do so. ${ }^{7}$ Older TCAs should be reserved for when first-line treatment has failed, and monoamine oxidase inhibitors should only be prescribed by experts. ${ }^{7}$

If patients have tried SSRIs for a prior episode without response, mirtazapine, lofepramine, nortriptyline, or an SNRI would be a reasonable first choice (venlafaxine or duloxetine rather than vortioxetine in the first instance). If they have had no response to previous treatment with SSRIs, mirtazapine, and SNRIs, then agomelatine would be a reasonable choice.

The BAP guidelines state that useful pharmacogenetic predictors of response to antidepressants are not available, and there is very limited evidence that past or family history is of use in predicting a differential response to different antidepressants. However, considering patients' preferences improves treatment adherence and may improve outcomes. ${ }^{7}$

Patients should usually be reviewed no later than 2 weeks after starting an antidepressant 11 week if aged under 30 years or thought to be at increased risk of suicide), as the risk of self-harm may be increased during the initiation of treatment. Subsequently, patients can be advised to adjust doses and dose timings themselves, with a further review at 4 weeks.

Randomised controlled trials tend to recruit highly selected patients without comorbidities, and usually summarise average treatment effects at the group level, rather than investigating potentially important modifiers of treatment response at the level of individual patients. ${ }^{1}$ It is important to emphasise, therefore, that there is significant inter-individual variation in tolerability and response to antidepressants, and a flexible, responsive approach is usually required to find the right treatment for a particular patient. However, effective treatment is a clinical imperative given the morbidity and mortality associated with major depressive disorder.

\section{REFERENCES}

1. Cipriani A, Furukawa TA, Salanti G, et al. Comparative efficacy and acceptability of 21 antidepressant drugs for the acute treatment of adults with major depressive disorder: a systematic review and network meta-analysis. Lancet 2018; 391(10128): 1357-1366.

2. Cipriani A, Furukawa TA, Salanti G, et al. Comparative efficacy and acceptability of 12 new-generation antidepressants: a multipletreatments meta-analysis. Lancet 2009; 373(9665): 746-758

3. Kendrick T, Stuart B, Newell C, et al. Antidepressants can benefit patients with major depression, and a 10th key issue is which drugs should be used first-line. Br J Gen Pract 2018; DOI: https://doi.org/10.3399/bjgp18X695681).

4. Regional Drug and Therapeutics Centre (Newcastle). Cost comparison charts November 2018. 2018. http://gmmmg.nhs.uk/docs/cost_ comparison_charts.pdf (accessed 4 Feb 2019).

5. Medicines and Healthcare products Regulatory Agency. Citalopram and escitalopram: QT interval prolongation. 2014. https://umw. gov.uk/drug-safety-update/citalopram-andescitalopram-qt-interval-prolongation laccessed 4 Feb 2019).

6. Kennedy SH, Andersen HF, Thase ME. Escitalopram in the treatment of major depressive disorder: a meta-analysis. Curr Med Res Opin 2009; 25(1): 161-175.

7. Cleare A, Pariante $\mathrm{CM}$, Young $\mathrm{AH}$, et al. Evidence-based guidelines for treating depressive disorders with antidepressants:

\section{ADDRESS FOR CORRESPONDENCE}

\section{Tony Kendrick}

Primary Care and Population Sciences, Aldermoor Health Centre, Aldermoor Close, Southampton

S016 5ST, UK.

\section{Email: ark1dsoton.ac.uk}

\section{atony_kendrick}

\section{Tony Kendrick}

Professor of Primary Care, Primary Care and Population Sciences, University of Southampton, Southampton.

\section{David Taylor}

Director of Pharmacy and Pathology, Maudsley Hospital, and Professor of Psychopharmacology, King's College London, London.

\section{Chris F Johnson,}

Antidepressant Specialist Pharmacist, NHS Greater Glasgow \& Clyde Pharmacy \& Prescribing Support Unit, Pharmacy Services, NHS Greater Glasgow \& Clyde, Glasgow.

\section{Provenance}

Commissioned; externally peer reviewed.

\section{Competing interests}

Tony Kendrick and David Taylor are members of the current NICE Guideline Development Group for the Depression in Adults guideline update. The views expressed are those of the authors and not necessarily those of NICE, the NHS, or the Department of Health.

\section{DOI: https://doi.org/10.3399/bjgp19X701405}

a revision of the 2008 British Association for Psychopharmacology guidelines. J Psychopharmacol 2015; 29(5): 459-525.

8. Taylor D, Sparshatt A, Varma S, Olofinjana O. Antidepressant efficacy of agomelatine: metaanalysis of published and unpublished studies. BMJ 2014; 348: g1888.

9. Koesters M, Ostuzzi G, Guaiana G, et al. Vortioxetine for depression in adults. Cochrane Database Syst Rev2017; (7): CD011520.

10. Prescribing and Medicines Team, NHS Digital. Prescription cost analysis - England, 2017. 2018. Table 5: Items by BNF chemicals 2007 to 2017. https://files.digital.nhs.uk/publication/g/r/ pca-eng-2007-17-trends-items.xlsx laccessed 4 Feb 2019).

11. Watanabe N, Omori IM, Nakagawa A, et al. Mirtazapine versus other antidepressive agents for depression. Cochrane Database Syst Rev 2011; (12): CD006528.

12. National Institute for Health and Care Excellence. Depression in adults: recognition and management. CG90. London: NICE, 2009, updated 2018. https://uww.nice.org.uk/ guidance/cg90 laccessed 4 Feb 2019).

13. Arroll B, Chin WY, Moir F, Dowrick C. An evidence-based first consultation for depression: nine key messages. Br J Gen Pract 2018; DOI: https://doi.org/10.3399/bjgp18X695681.

14. Johnson CF, Williams B, MacGillivray SA, et al 'Doing the right thing': factors influencing GP prescribing of antidepressants and prescribed doses. BMC Fam Pract 2017; 18(1): 72. 